\title{
The differences between the horizontal membership function used in multidimensional fuzzy arithmetic and the inverse membership function used in gradual arithmetic
}

\author{
Andrzej Piegat ${ }^{1} \cdot$ Marcin Pluciński ${ }^{1}$ (D) \\ Received: 15 July 2021 / Accepted: 28 September 2021 / Published online: 8 October 2021 \\ (c) The Author(s) 2021
}

\begin{abstract}
In the last few years, the number of applications of the multidimensional fuzzy arithmetic (MFA) and the multidimensional interval arithmetic is expanding. Authors of new papers about applications of MFA are often faced with comments from other researchers, especially the gradual arithmetic (GA) proponents, that the horizontal membership function (HMF) used in MFA is the same as the inverse membership function (InvMF) used in GA, and that MFA itself adds nothing new to the fuzzy arithmetic. This view leads to unfair evaluations of scientific papers about MFA applications submitted to scientific journals and to unnecessary disagreements between MFA and GA proponents. The purpose of this paper is to carefully analyze the two types of functions (HMF and InvMF) and to demonstrate their important differences. The basic and decisive difference is the dimensionality of both functions, which is illustrated by examples. It should also be added that HMF has proven its usefulness in solving difficult problems such as: systems of fuzzy equations or fuzzy differential equations, which is confirmed by numerous publications. The paper enable the reader to have a deeper understanding of the multidimensional fuzzy arithmetic.
\end{abstract}

Keywords Fuzzy arithmetic · Fuzzy calculus · Multidimensional fuzzy arithmetic · Gradual arithmetic $\cdot$ Horizontal membership function · Inverse membership function

\section{Introduction}

The concept of a horizontal membership function (HMF) is used in the multidimensional fuzzy arithmetic (MFA) (Piegat and Landowski 2013, 2015; Piegat and Pluciński 2015, 2017), which in first publications was called RDM fuzzy arithmetic (from relative-distance-measure). MFA due to its multidimensional approach to fuzzy calculations is more difficult to understand than 2D types of the fuzzy arithmetic (FA). Therefore, MFA requires a thorough

Andrzej Piegat and Marcin Pluciński are contributed equally to this work.

Marcin Pluciński

mplucinski@wi.zut.edu.pl

Andrzej Piegat

apiegat@wi.zut.edu.pl

1 Faculty of Computer Science and Information Systems, West

Pomeranian University of Technology, Żołnierska 49,

71-210 Szczecin, Poland explanation of its essence and details, supported by examples. The scientific team studying MFA has carefully explained how to perform each arithmetic operation in successive papers. Piegat and Landowski (2013) present the implementation of multiplication and division operations, Piegat and Pluciński (2015) present addition, and Piegat and Pluciński (2017) present the division operation, which is slightly more difficult than the other arithmetic operations. Piegat and Landowski (2015) presents the first applications of HMF.

An important advantage of using MFA is its ability to find algebraic solutions of equations, including systems of equations with multiple unknowns. Solving fuzzy equations is very difficult and there are many publications on this subject. Kołodziejczyk et al. (2020) show the advantage of HMF and MFA over one of the methods of solving nonlinear fuzzy equations known in the literature.

Solving differential equations is also a very difficult problem-there are a lot of publications in this area as well. The application of HMF and MFA to this task allowed to obtain precise solutions of differential 
equations, which are very important in the modeling, study and control of dynamic systems. Mazandarani and Zhao (2019), Najariyan and Zhao (2020) and Mazandarani and Xiu (2021) present the results of this type of research.

This paper compares HMF used in MFA and the inverse membership function (InvMF) used to create gradual numbers (GN) used in the gradual interval arithmetic (GIA). GIA can be considered somewhat competitive to MFA. The concept of gradual elements of fuzzy sets was introduced by Dubois and Prade (2008), and the concept of gradual numbers and the fundamentals of GIA were presented by Fortin et al. (2008). Currently, GIA is being developed by, among others (Boukezzoula et al. 2021). An extended notion of GIA - thick gradual intervals, which is equivalent to type-2 fuzzy intervals used in the fuzzy arithmetic, has also been developed (Boukezzoula et al. 2019). In turn, Piegat and Landowski (2021a) present a new MFA based on type-2 HMFs which are extensions of type-1 HMFs. Application examples are also presented in this paper.

The fact, that MFA is more difficult to understand than 2D types of the fuzzy arithmetic has sometimes resulted in erroneous comments about it expressed in some FA papers and in opinions of some reviewers. One of the objections against MFA is the assumption that HMF used in MFA and and inverse membership function (InMF) used in the gradual interval arithmetic (GIA) (Boukezzoula et al. 2021; Dubois and Prade 2008; Fortin et al. 2008) are one and the same. More precisely, that HMF in the theory of gradual numbers is called the assignment function (AF) of a gradual number. The authors of this objection also propose that HMF should be treated as the inverse membership function.

Such opinions have not only theoretical, but also practical implications. Authors of publications on various applications of MFA and HMF inform us about erroneous comments made in reviews of their papers that lead to an unfair evaluation. Erroneous opinions about MFA and HMF are also shared either verbally or by correspondence. Therefore, a strong motivation for the preparation of this paper is to prevent the spread of misinformation and the downgrading of HMF and MFA.

The main contribution of this paper is the detailed analysis of the properties of InvMF used in GIA and HMF used in HMA and the identification of the differences between them. It can be noted at the very beginning that the basic and main difference between InvMF and HMF is the different dimensionality of these two functions resulting from their nature. The higher dimensionality of HMF provides advantages in solving difficult FA problems such as solving systems of equations (Kołodziejczyk et al. 2020), solving fuzzy differential equations (Mazandarani and Zhao 2019; Najariyan and Zhao 2020; Mazandarani and Xiu 2021), implementing operations in type-2 arithmetic (Piegat and Landowski 2021a, b).

In the paper, we want to make it clear that HMF and InvMF are not the same. In Sect. 2, we will explain what HMF is and how it is used in MFA. Sect. 3 will explain the concept of an assignment function, InvMF, a gradual number (GN), a gradual interval (GI) and will show how these terms are used in GIA. Section 4 will explain the differences between HMF and the assignment function. Section 5 will give examples of calculations using HMFs and InMFs and Sect. 6 presents conclusions.

Notations used in the paper: AF-assignment function, CI-constrained interval, FI-fuzzy interval, FN-fuzzy number, FS - fuzzy set, GA-gradual arithmetic, GIAgradual interval arithmetic, GN-gradual number, MFmembership function, HMF-horizontal membership function, IA - interval arithmetic, InvMF-inverse membership function, MFA-multidimensional fuzzy arithmetic, RDM-relative-distance-measure, RDM-FARDM-fuzzy arithmetic, SIA — standard interval arithmetic.

\section{Horizontal membership function}

Figure 1 shows a trapezoidal MF defined by a quadruple $\left(a_{1}, a_{2}, a_{3}, a_{4}\right)$. This MF presents the knowledge about the value of the variable $a$ that is not known exactly.

The traditional, most commonly used, vertical MF (VMF) $\mu(a)$ is defined by the formula (1).

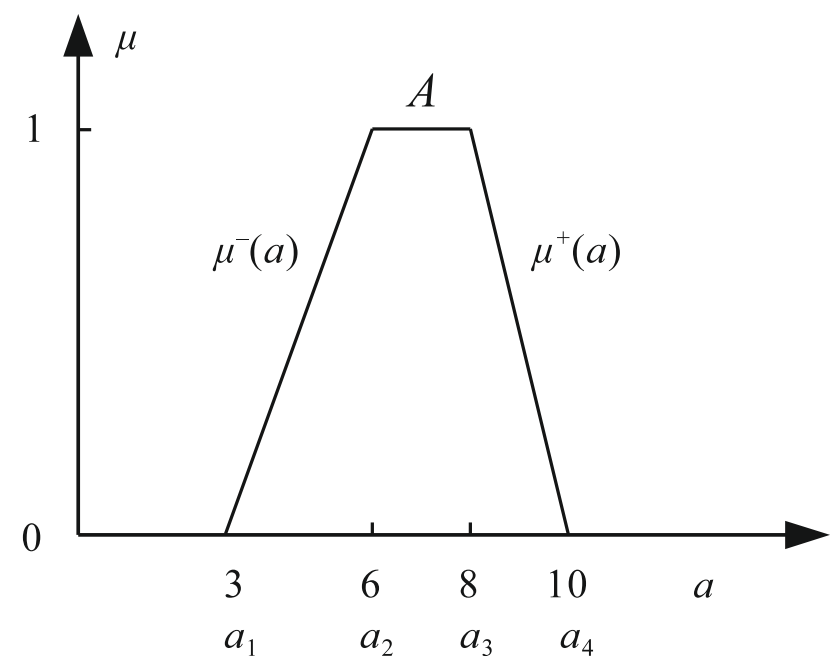

Fig. 1 The trapezoidal membership function is defined by quadruple $\left(a_{1}, a_{2}, a_{3}, a_{4}\right)=(3,6,8,10)$, where $\mu^{-}(a)$ means the left border and $\mu^{+}(a)$ the right border of MF 


$$
\mu(a)=\left\{\begin{array}{llr}
\text { for } & a \in\left[a_{1}, a_{2}\right] & \mu(a)=\mu^{-}(a)=\frac{a-a_{1}}{a_{2}-a_{1}} \\
\text { for } & a \in\left[a_{2}, a_{3}\right] & \mu(a)=1 \\
\text { for } & a \in\left[a_{3}, a_{4}\right] & \mu(a)=\mu^{+}(a)=\frac{a_{4}-a}{a_{4}-a_{3}}
\end{array}\right.
$$

This MF corresponds to the first definition of the fuzzy set (FS) by Zadeh (1965). It defines borders of FS and for each value of the variable $a$ it assigns the degree of membership $\mu(a)$ as the output. On the basis of vertical models of the left $\mu^{-}(a)$ and right $\mu^{+}(a)$ borders, it is possible to calculate inverse functions $a^{-}(\mu)$ and $a^{+}(\mu)$ defined by the formula (2).

$a^{-}(\mu)=a_{1}+\mu\left(a_{2}-a_{1}\right), \quad \mu \in[0,1]$

$a^{+}(\mu)=a_{4}-\mu\left(a_{4}-a_{3}\right), \quad \mu \in[0,1]$

In inverse functions, the informational output is not the degree of membership $\mu$ but the value of the variable $a$, Fig. 2.

RDM-variable $\gamma_{a}$ specifies a set of points that lay at an equal, relative distance from the left border $a^{-}(\mu)$. Figure 2 shows the exemplary set that is located at the distance $\gamma_{a}=0.5$ from the left border. The RDM-variable allows to enter the same (normalized to the range $[0,1]$ ) distance of a point located on any $\alpha$-cut of MF. Using the concept of the RDM-variable it is possible to define HMF (3) for the fuzzy set $A$.

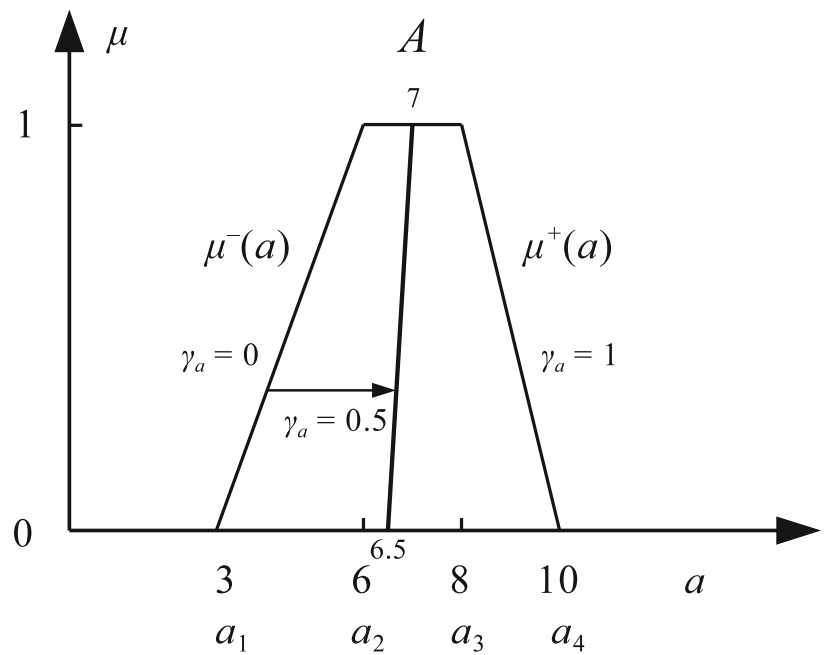

Fig. 2 Notations used in HMF: $\gamma_{a}-\mathrm{RDM}$-variable, $a^{-}(\mu)$-left border, and $a^{+}(\mu)$-right border

$$
\begin{aligned}
& A: a \in A \\
& a\left(\mu, \gamma_{a}\right)=a^{-}(\mu)+\gamma_{a}\left[a^{+}(\mu)-a^{-}(\mu)\right], \quad \mu, \gamma_{a} \in[0,1] \\
& a\left(\mu, \gamma_{a}\right)=a_{1}+\mu\left(a_{2}-a_{1}\right) \\
& +\gamma_{a}\left[a_{4}-a_{1}-\mu\left(a_{4}-a_{3}-a_{2}+a_{1}\right)\right], \\
& \mu, \gamma_{a} \in[0,1]
\end{aligned}
$$

The formula (3) looks complicated, but after substituting the numerical values of coefficients given in Fig. 1, it is simplified to the formula (4).

$A: a \in A$

$a\left(\mu, \gamma_{a}\right)=3+3 \mu+\gamma_{a}[7-5 \mu], \quad \mu, \gamma_{a} \in[0,1]$

Formulas (3) and (4) defining HMF allow to specify each point lying inside the fuzzy set $A$ with the coordinates $\left(\mu, \gamma_{a}\right)$. For example at the level $\mu=0.5$ and $\gamma_{a}=0.5$, there is a point with the coordinate $a=6.75$, Fig. 2. Since the relation $\mu, \gamma_{a} \in[0,1]$ is valid, $\operatorname{HMF}$ (3) is a mathematical model not only of borders of MF, but also of its entire interior (body) (Fig. 3).

The HMF shown in Fig. 3 results from the second definition of the fuzzy set of Zadeh (1975), according to which FS can be composed of its horizontal $\alpha$-cuts. Modeling of the entire area of FS is possible only due to the fact that in HMF (3) the value of the variable $a\left(\mu, \gamma_{a}\right)$ depends not on one but on 2 variables: $\mu$ and $\gamma_{a}$. This means that HMF exists in 3D-space, Fig. 4. Figure 3 shows only a projection of this function from $3 \mathrm{D}$ to a $2 \mathrm{D}$-space.

HMFs, unlike VMFs, are particularly suitable for performing arithmetic operations, which are simply performed on values of variables $a$ and $b$ that occur in such operations. In the case of HMFs, the objection is sometimes raised that the membership function should be a function having the degree of membership $\mu(a)$ as the output and that the output should not be the value of the variable $a$ itself. In opinion of authors, this objection is rather incorrect,

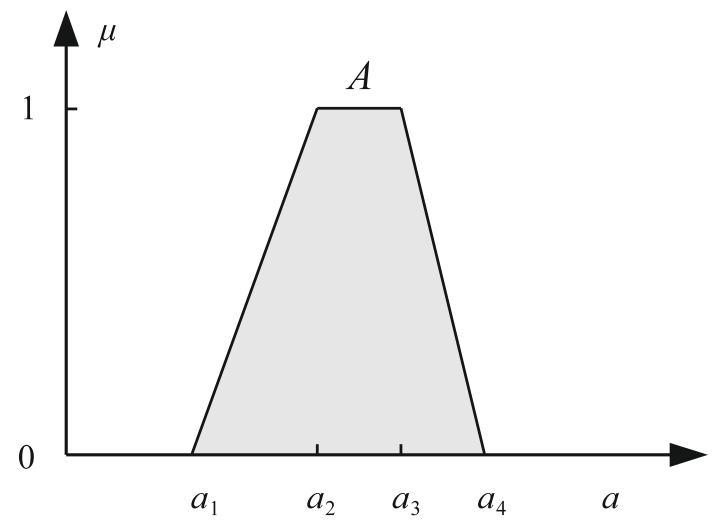

Fig. 3 Illustration of the modeling by HMF (3) of the entire area of the fuzzy set $A$ together with its borders 


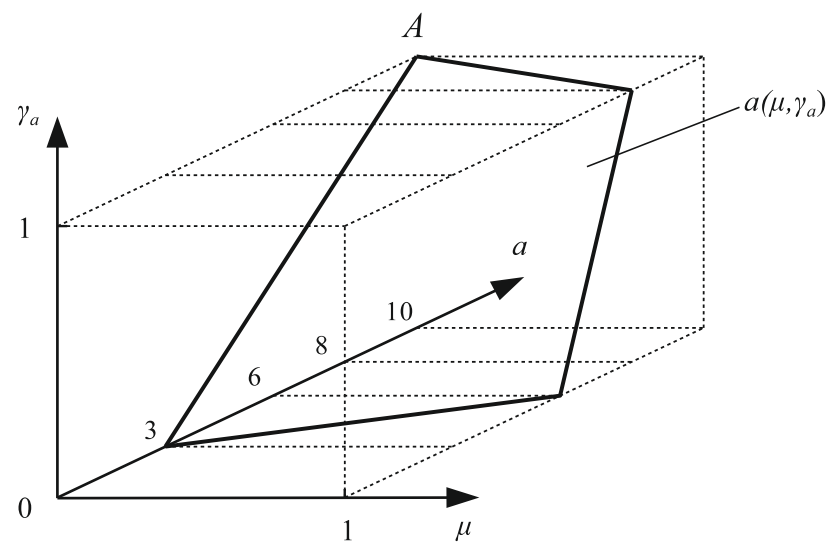

Fig. $4 \mathrm{HMF}$ of the fuzzy set $A$ in the full 3D-space $a \times \mu \times \gamma_{a}$

because MF can also be interpreted as a function providing information about the set of values of the variable $a$ belonging to the fuzzy set under consideration and describing the $\alpha$-cut of this set at the level $\mu=\alpha$. For example: HMF (4) from Fig. 2 provides information that at the level $\alpha=0.5$, the set $A$ includes $\alpha$-cut (interval) $[4.5,9]$.

Using HMFs of fuzzy numbers, we can perform arithmetic operations according to the formula (5).

$A: a \in A, \quad B: b \in B$

$a=a\left(\mu, \gamma_{a}\right), \quad b=b\left(\mu, \gamma_{b}\right), \quad \mu, \gamma_{a}, \gamma_{b} \in[0,1]$

$A \odot B=X, \quad X: x \in X, \odot \in\{+,-, \times, \div\}$

$a\left(\mu, \gamma_{a}\right) \odot b\left(\mu, \gamma_{b}\right)=x\left(\mu, \gamma_{a}, \gamma_{b}\right)$

The algebraic result $x\left(\mu, \gamma_{a}, \gamma_{b}\right)$ exists in the 4D-space and it is an universal result, i.e. one that satisfies all possible forms of the mathematical formula in which it occurs. For example, for addition $A+B=X$, the result $X$ satisfies the following forms: $A+B=X, A=X-B, B=X-A$, $A+B-X=0$. When performing complex mathematical calculations that are realized in parts, only multidimensional intermediate results should be inserted into the final formula and not the spans of these intermediate results, as it is done today. The span $\operatorname{SP}_{x}(\mu)$ is just a simplified twodimensional information about the full multidimensional result. The span $S P_{x}(\mu)$ is calculated using the formula (6).

$X: x\left(\mu, \gamma_{a}, \gamma_{b}\right) \in X$

$\mathrm{SP}_{x}(\mu)=\left[\min _{\gamma_{a}, \gamma_{b}} x\left(\mu, \gamma_{a}, \gamma_{b}\right), \max _{\gamma_{a}, \gamma_{b}} x\left(\mu, \gamma_{a}, \gamma_{b}\right)\right]$

Further information about performing arithmetic operations on FN with HMFs can be found in authors' publications (Piegat and Landowski 2013, 2015; Piegat and Pluciński 2015, 2017).

\section{Assignment function used in gradual numbers and the gradual arithmetic}

As noted in Sect. 1, it is sometimes alleged that HMF used in MFA is the same as InvMF, also referred to as the assignment function (AF), used in the gradual interval arithmetic (GIA). Therefore, it is necessary to explain exactly what $\mathrm{AF}$ is. In publications (Boukezzoula et al. 2021; Dubois and Prade 2008; Fortin et al. 2008) their authors presented the concept of GIA, in which gradual intervals (GIs) are used, which contain gradual numbers (GNs) as their components. Detailed definitions of above terms and various explanations related to them are presented by (Boukezzoula et al. 2021; Dubois and Prade 2008; Fortin et al. 2008). In this paper, the GIA concept will be presented only briefly and we will use the original notations from cited papers. The authors of the GIA concept claim that imprecise value (which in FA is called a fuzzy number) should rather be called a fuzzy interval (FI) (Fortin et al. 2008). According to them, FIs are a subclass of GIs, which are a broader concept, because the left border of GI does not have to be a non-decreasing function and the right border does not have to be a non-increasing function, as is the case of FIs. Hence, every FI is GI but not every GI is FI. Figure 5 shows a trapezoidal and a triangular FI which is also GI.

The fuzzy interval $(\mathrm{FI}=\mathrm{GI})$ has left and right borders being gradual numbers (GNs) by definition. "A gradual number allows to look at fuzzy intervals as crisp intervals on the set of gradual real numbers" (Fortin et al. 2008). Such an interpretation of GIs enables the application of all existing methods of interval arithmetic (IA) in GIA. Boukezzoula et al. (2021) list the following methods of IA:

1. Standard interval arithmetic (SIA).

2. Instantiated interval arithmetic (IIA).

3. Extended interval arithmetic (EIA).

4. Non-standard interval arithmetic (NIA).

5. Generalized Hukuhara interval arithmetic (GHIA).

6. Optimistic interval arithmetic (OIA).

7. Constrained interval arithmetic (CIA).

8. Single level constrained interval arithmetic (SCIA).

9. Requisite constrained interval arithmetic (RCIA).

In the case of FIs shown in Fig. 5, their left and right borders can be described using traditional vertical MFs given by formulas (7) (Fortin et al. 2008):

$\mu_{M}^{-}(m)=\frac{m-m_{0}^{-}}{m_{1}^{-}-m_{0}^{-}}, \quad \mu_{M}^{+}(m)=\frac{m_{0}^{+}-m}{m_{0}^{+}-m_{1}^{+}}$.

The input information in the formula (7) are values of the variable $m$ and the output information are degrees of membership $\mu^{-}(m)$ and $\mu^{+}(m)$. From vertical MFs (7) we 
Fig. 5 Trapezoidal and triangular fuzzy interval which is also a gradual interval: their left and right borders are gradual numbers

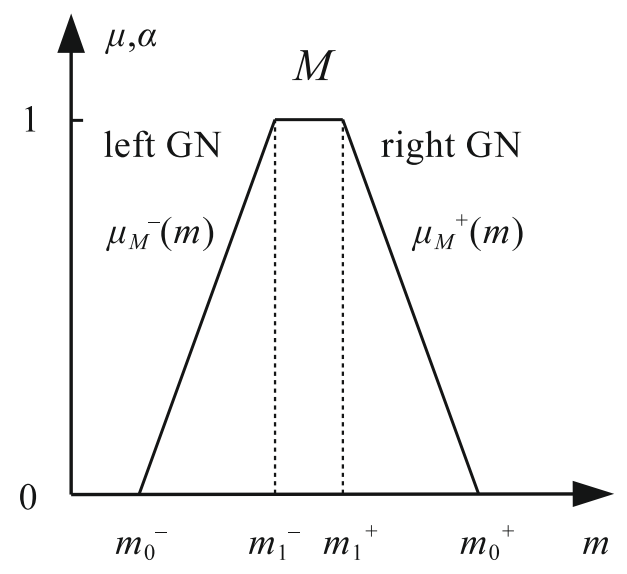

can determine InvMFs (8), where $\alpha$ denotes the degree of membership $\mu$ ( $\alpha$-cuts).

$$
\begin{array}{ll}
\left(\mu_{M}^{-}\right)^{-1}=m^{-}=m_{0}^{-}+\alpha\left(m_{1}^{-}-m_{0}^{-}\right), & \alpha \in(0,1] \\
\left(\mu_{M}^{+}\right)^{-1}=m^{+}=m_{0}^{+}+\alpha\left(m_{0}^{+}-m_{1}^{+}\right), & \alpha \in(0,1]
\end{array}
$$

MFs (8) can be called inverse MFs, because the output information here is the value of the variable $m$ corresponding to the membership degree $\alpha$. In the case of FIs, each border, left or right, corresponds to only one InvMF (in the case of GIs there may be more) and then the whole function can be defined as the "assignment function" (Fortin et al. 2008). "The assignment function $A_{\tilde{r}}$, for each value $\alpha \in(0,1]$ assigns a real number $r=A_{\tilde{r}}(\alpha)$." AFs allow to define borders of FIs, which (Fortin et al. 2008) call "gradual real numbers". The definition of the gradual number is as follows. "A gradual real number $r$ is defined by an assignment function $A_{\tilde{r}}$ from $(0,1]$ (the unit interval minus 0 ) to the reals." Inverse functions $\left(\mu_{M}^{-}\right)^{-1}$ and $\left(\mu_{M}^{+}\right)^{-1}$ are special cases of GNs. Figure 6 shows 2 examples of GNs.

When performing arithmetic operations on FIs in accordance with the rules of GIA, calculations are performed on borders of FIs, i.e. on GNs. Further on, definitions of addition and multiplication of GNs (Fortin et al. 2008) will be given.

\subsection{Addition of GNs}

The sum of GNs $\tilde{r}$ and $\tilde{s}$ is defined by summing their assignment functions. For $\tilde{r}+\tilde{s}$ and $\alpha \in(0,1]$ we have:

$A_{\tilde{r}+\tilde{s}}(\alpha)=A_{\tilde{r}}(\alpha)+A_{\tilde{s}}(\alpha)$.

An example of adding GNs from Fig. 6 is given by the formula (10).

$$
\begin{aligned}
A_{\tilde{r}} & =r_{0}+\alpha\left(r_{1}-r_{0}\right)=1+\alpha, \quad \alpha \in(0,1] \\
A_{\tilde{s}} & =s_{0}+\alpha\left(s_{1}-s_{0}\right)=3+2 \alpha, \quad \alpha \in(0,1] \\
A_{\tilde{r}+\tilde{s}} & =A_{\tilde{r}}+A_{\tilde{s}}=\left(r_{0}+s_{0}\right)+\alpha\left[\left(r_{1}-r_{0}\right)+\left(s_{1}-s_{0}\right)\right] \\
& =4+3 \alpha, \quad \alpha \in(0,1]
\end{aligned}
$$

The result of adding is GN with a shape similar to $\tilde{r}$ and $\tilde{s}$ (Fig. 6), but with a wider support. The subtraction operation can be realized as adding $\tilde{r}+(-\tilde{s})$.
Fig. 6 An example of two gradual numbers $\tilde{r}$ and $\tilde{s}$ with their assignment functions $A_{\tilde{r}}$ and $A_{\tilde{s}}$
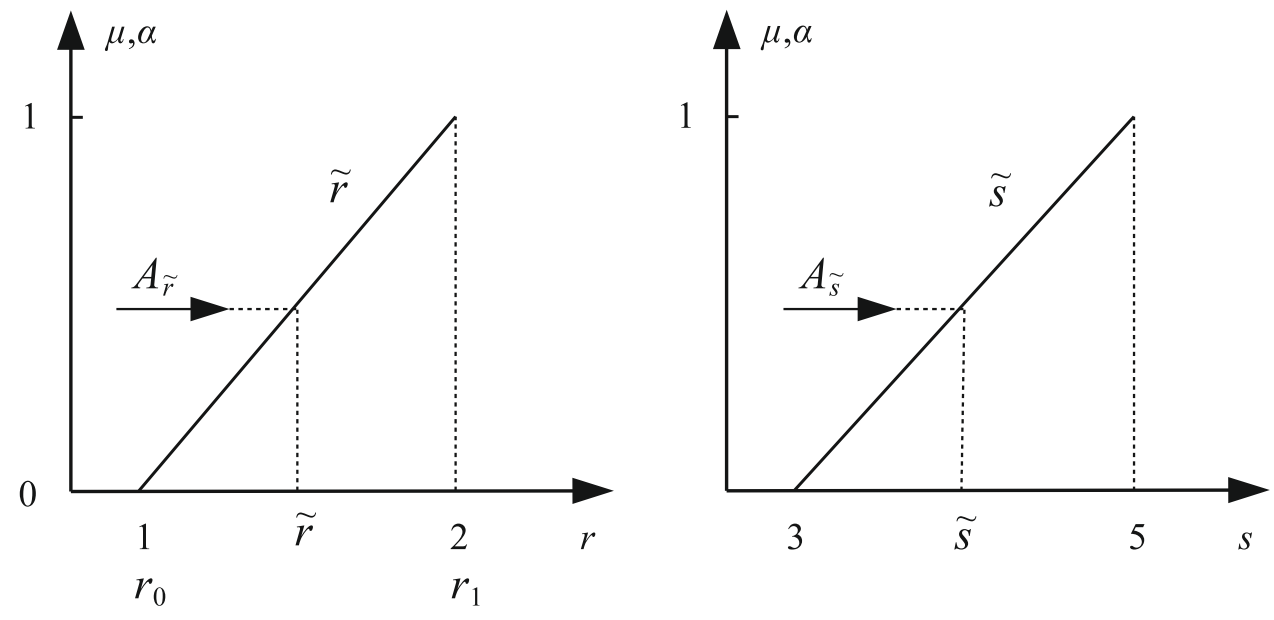


\subsection{Multiplication of GNs}

The product and the quotient of GNs can be defined in a similar way (with caution when dividing by a gradual number $\tilde{r}$ such that $A_{\tilde{r}}(\alpha)=0$ for some $\alpha$ ). If two GNs $\tilde{r}$ and $\tilde{s}$ are given (10), then their product $A_{\tilde{r} \tilde{s}}$ is determined by the formula (11).

$$
\begin{aligned}
A_{\tilde{r} \tilde{s}}(\alpha) & =\left[r_{0}+\alpha\left(r_{1}-r_{0}\right)\right]\left[s_{0}+\alpha\left(s_{1}-s_{0}\right)\right] \\
& =(1+\alpha)(3+2 \alpha), \quad \alpha \in(0,1]
\end{aligned}
$$

The operation $A_{\tilde{r}} / A_{\tilde{s}}$ can be performed as a multiplication operation $A_{\tilde{r}}\left(1 / A_{\tilde{s}}\right)$.

\subsection{Gradual interval arithmetic}

Knowing the arithmetic of GNs, it is now possible to present the arithmetic of FIs based on GIA principles (FI is a special case of GI). In the GIA arithmetic operations are performed similarly to the interval arithmetic, using for calculations one of existing types of the interval arithmetic. Boukezzoula et al. (2021) describes this principle as follows. "If borders of conventional interval $[a]=\left[a^{-}, a^{+}\right]$are replaced by assignment functions $a^{-}(\alpha)$ and $a^{+}(\alpha)$ representing two GNs, we get gradual interval $[a(\alpha)]=$ $\left[a^{-}(\alpha), a^{+}(\alpha)\right]$ which borders are used in interval arithmetic operations". Figure 7 shows two FIs that will be used in the addition example.

Fuzzy interval $[a(\alpha)]$ is defined by two border GNs represented by two assignment functions $a^{-}(\alpha)$ and $a^{+}(\alpha)$ given by (12).

$$
\begin{aligned}
{[a(\alpha)] } & =\left[a^{-}(\alpha), a^{+}(\alpha)\right] \\
& =[1+2 \alpha, 6-2 \alpha], \quad \alpha \in(0,1]
\end{aligned}
$$

Similarly, FI $[b(\alpha)]$ is given by (13).

$$
\begin{aligned}
{[b(\alpha)] } & =\left[b^{-}(\alpha), b^{+}(\alpha)\right] \\
& =[5+2 \alpha, 9-2 \alpha], \quad \alpha \in(0,1]
\end{aligned}
$$

The sum of intervals $[a(\alpha)]+[b(\alpha)]=[x(\alpha)]$ is given by the formula (14) and shown in Fig. 8.

$$
\begin{aligned}
{[a(\alpha)]+[b(\alpha)] } & =[1+2 \alpha, 6-2 \alpha]+[5+2 \alpha, 9-2 \alpha] \\
& =[6+4 \alpha, 15-4 \alpha], \quad \alpha \in(0,1]
\end{aligned}
$$

Note, that adding FIs $[a(\alpha)]$ and $[b(\alpha)]$ using GIA resulted in a fuzzy interval $[x(\alpha)]$ containing not one but two assignment functions. Next, we will see if it is the same in calculations using HMFs.

\section{Comparison of the assignment function used in GIA and horizontal membership function used in MFA}

There are differences between AF and HMF which will be presented now. The mathematical model of the fuzzy interval used in GIA consists of two assignment functions $a^{-}(\alpha)$ and $a^{+}(\alpha)$ :

$[a(\alpha)]=\left[a^{-}(\alpha), a^{+}(\alpha)\right], \quad \alpha \in(0,1]$.

In the case of the trapezoidal fuzzy interval from Fig. 7, it takes the form (16).

$[a(\alpha)]=[1+2 \alpha, 6-2 \alpha], \quad \alpha \in(0,1]$

The model $[a(\alpha)]$ is 2 -dimensional, see Fig. 7. In the case of MFA, the model of FI is called HMF and is 3-dimensional, (17).

$$
\begin{aligned}
& A: \quad a \in A \\
& \quad a\left(\alpha, \gamma_{a}\right)=a^{-}(\alpha)+\gamma_{a}\left[a^{+}(\alpha)-a^{-}(\alpha)\right] \\
& \quad=(1+2 \alpha)+\gamma_{a}(5-4 \alpha) \quad \alpha, \gamma_{a} \in[0,1]
\end{aligned}
$$

Fig. 7 Fuzzy intervals $[a(\alpha)]$ and $[b(\alpha)]$ used in the addition operation $[a(\alpha)]+[b(\alpha)]$
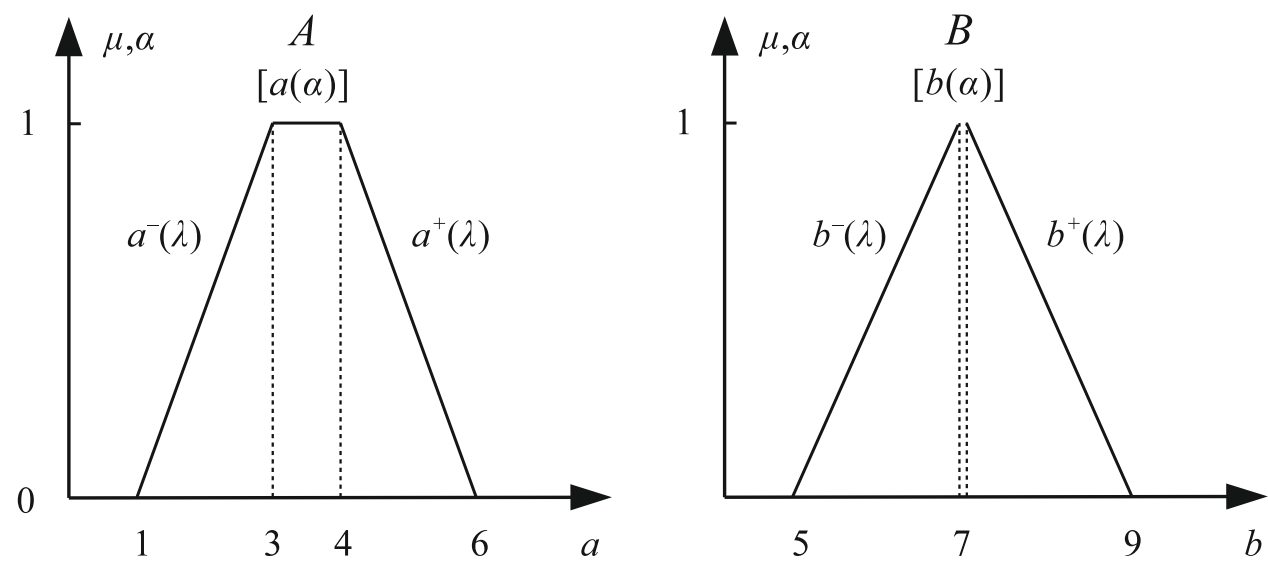


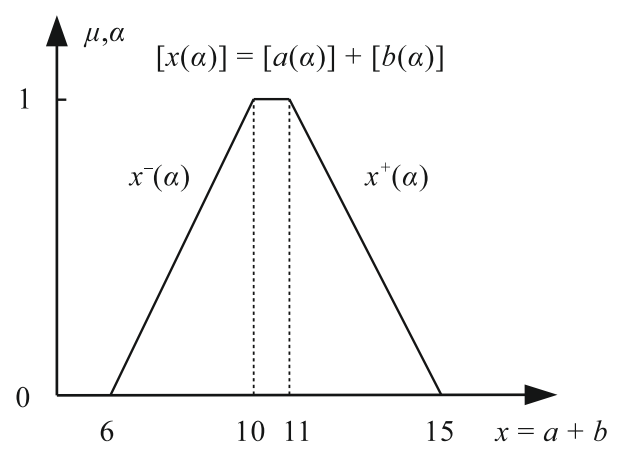

Fig. 8 Result of the addition of two fuzzy intervals from Fig. 7 using the gradual interval arithmetic based on assignment functions

The difference between the two mathematical models is illustrated in Fig. 9. Gradual interval $[a(\alpha)]$ represents only borders $a^{-}(\alpha)$ and $a^{+}(\alpha)$ of the fuzzy interval. For this reason, only borders are used in arithmetic calculations. However, the interior of FI between these borders is not included in calculations. This affects calculation results in many cases. Using HMF, which represents both borders and the interior of FI, we obtain sometimes identical and sometimes different results than in the case of using gradual interval models $[a(\alpha)]$. Examples illustrating this fact can be found in publications of the authors on MFA. Examples will also be given in the next section to illustrate the difference in results of both methods. In gradual arithmetic, borders (AFs) of FI $a^{-}(\alpha)$ and $a^{+}(\alpha)$ take part in arithmetic operations separately. In the case of HMFs, these borders are combined in a uniform function $a\left(\alpha, \gamma_{a}\right)=a^{-}(\alpha)+\gamma_{a}\left[a^{+}(\alpha)-a^{-}(\alpha)\right]$ forming a geometrically single whole and only this unity takes part in calculations. This has a significant impact on obtained results.

\section{Examples illustrating differences in results obtained with the use of gradual interval and horizontal membership function models}

First example will consist in solving the equation $A+X=B$, where fuzzy intervals $A$ and $B$ represent the knowledge about the uncertain values of the variables $a$ and $b$. The intervals $[a(\alpha)]$ and $[b(\alpha)]$ are shown in Fig. 7 . These intervals have the following form: $[a(\alpha)]=$ $\left[a^{-}(\alpha), a^{+}(\alpha)\right]=[1+2 \alpha, 6-2 \alpha] \quad$ and $\quad[b(\alpha)]=\left[b^{-}(\alpha)\right.$, $\left.b^{+}(\alpha)\right]=[5+2 \alpha, 9-2 \alpha]$. Interval insides include AFs of left and right borders of intervals. First, the equation will be solved using GIA, and SIA of Warmus-Sunaga-Moore will be used as the interval arithmetic. It should be noted that in the case of GIA it is assumed that the solution of the equation is $2 \mathrm{D}$ gradual interval $[x(\alpha)]=\left[x^{-}(\alpha), x^{+}(\alpha)\right]$, i.e. the same mathematical form as in the case of intervals $\left[a^{-}(\alpha), a^{+}(\alpha)\right]$ and $\left[b^{-}(\alpha), b^{+}(\alpha)\right]$.

$$
\begin{aligned}
& {\left[a^{-}(\alpha), a^{+}(\alpha)\right]+\left[x^{-}(\alpha), x^{+}(\alpha)\right]=\left[b^{-}(\alpha), b^{+}(\alpha)\right]} \\
& {[1+2 \alpha, 6-2 \alpha]+\left[x^{-}(\alpha), x^{+}(\alpha)\right]=[5+2 \alpha, 9-2 \alpha]}
\end{aligned}
$$

Solving Eq. (18) according to the SIA rules, we obtain two equations (19).

$$
\begin{aligned}
& (1+2 \alpha)+x^{-}(\alpha)=(5+2 \alpha) \\
& (6-2 \alpha)+x^{+}(\alpha)=(9-2 \alpha)
\end{aligned}
$$

The solution of Eq. (19) is $x^{-}(\alpha)=4$ and $x^{+}(\alpha)=3$, which is the improper interval $[x(\alpha)]=\left[x^{-}(\alpha), x^{+}(\alpha)\right]=[4,3]$. This 'solution' means that there is no real value of $x$ satisfying equation (18), because the value of $x$ would have to satisfy the condition $x \geq 4$ and $x \leq 3$, what is impossible. However, let's check, using the common sense method of Instance Testing, whether there is actually any solution to the equation under study. At the level $\alpha=0$ the values $a=1$ and $b=9$ are possible,
Fig. 9 Illustration of the difference between the gradual interval membership function $[a(\alpha)]$ and the horizontal membership function $a\left(\alpha, \gamma_{a}\right)$
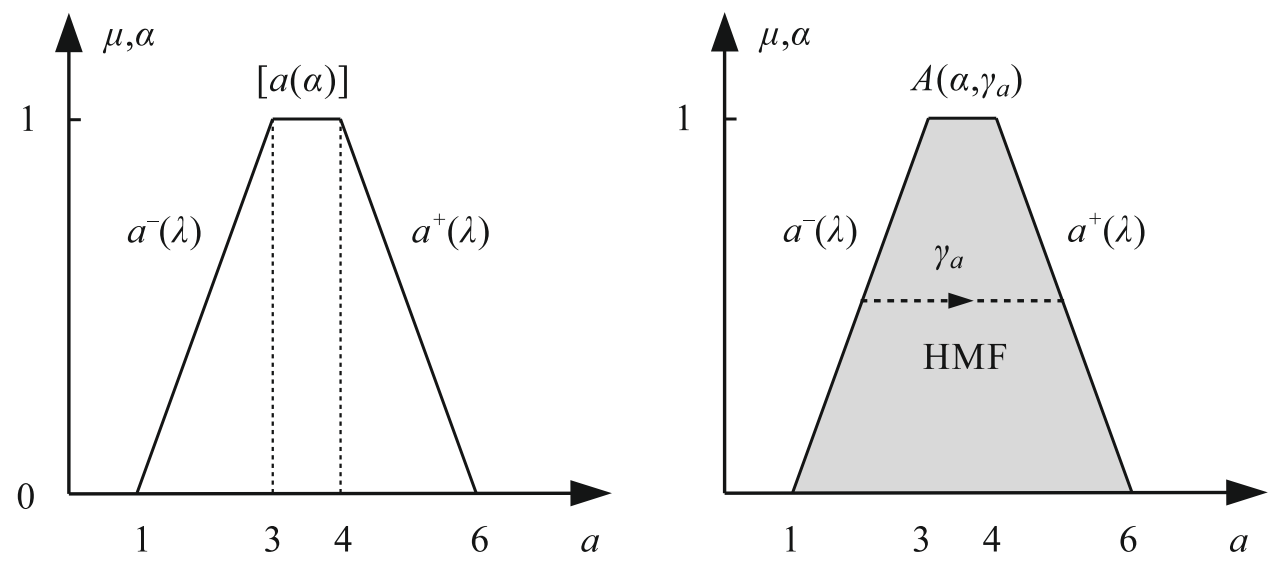
resulting in $x=b-a=8$. Hence, at least one instance solution is possible: $(a, b, x=b-a)=(1,9,8)$. We will see further that there are not only this one, but an infinite number of other possible solutions of the equation under study. For this purpose, MFA and HMFs will be used. The formulation of the task in terms of MFA is given by the formula (20).

$A: a\left(\alpha, \gamma_{a}\right) \in A, \quad B: b\left(\alpha, \gamma_{b}\right) \in B$

$A+X=B$

$X: x\left(\alpha, \gamma_{a}, \gamma_{b}\right) \in X$

$a\left(\alpha, \gamma_{a}\right)=(1+2 \alpha)+\gamma_{a}(5-4 \alpha), \quad \alpha, \gamma_{a} \in[0,1]$

$b\left(\alpha, \gamma_{b}\right)=(5+2 \alpha)+\gamma_{b}(4-4 \alpha), \quad \alpha, \gamma_{b} \in[0,1]$

$x\left(\alpha, \gamma_{a}, \gamma_{b}\right)=b\left(\alpha, \gamma_{b}\right)-a\left(\alpha, \gamma_{a}\right)$

$$
=4-\gamma_{a}(5-4 \alpha)+\gamma_{b}(4-4 \alpha)
$$

The solution of the problem is the set $(A, B, X=B-A)$ of triples $(a, b, x=b-a)$ defined by the formula (21).

$$
\begin{aligned}
(A, B, X=B-A) & =\left\{a\left(\alpha, \gamma_{a}\right), b\left(\alpha, \gamma_{b}\right), x\left(\alpha, \gamma_{a}, \gamma_{b}\right)\right. \\
& \left.=b\left(\alpha, \gamma_{b}\right)-a\left(\alpha, \gamma_{a}\right) \mid \alpha, \gamma_{a}, \gamma_{b} \in[0,1]\right\}
\end{aligned}
$$

The variable $x\left(\alpha, \gamma_{a}, \gamma_{b}\right)$ is a result variable that exists in 4D-space and cannot be seen directly. However, it is possible to determine and see the span $S P_{x}$ of this variable defined by the formula (22).

$S P_{x}=\left[\min _{\gamma_{a}, \gamma_{b}} x\left(\alpha, \gamma_{a}, \gamma_{b}\right), \max _{\gamma_{a}, \gamma_{b}} x\left(\alpha, \gamma_{a}, \gamma_{b}\right)\right], \quad \gamma_{a}, \gamma_{b} \in[0,1]$,

where: $\quad x\left(\alpha, \gamma_{a}, \gamma_{b}\right)=4-\gamma_{a}(5-4 \alpha)+\gamma_{b}(4-4 \alpha), \quad$ see formula (20).

Minimum $x\left(\alpha, \gamma_{a}, \gamma_{b}\right)$ is obtained for $\gamma_{a}=1, \gamma_{b}=0$ and maximum for $\gamma_{a}=0, \gamma_{b}=1$. Finally, the span of the result variable is given by the formula (23) and its visualization is shown in Fig. 10.

$S P_{x}=[-1+4 \alpha, 8-4 \alpha]$

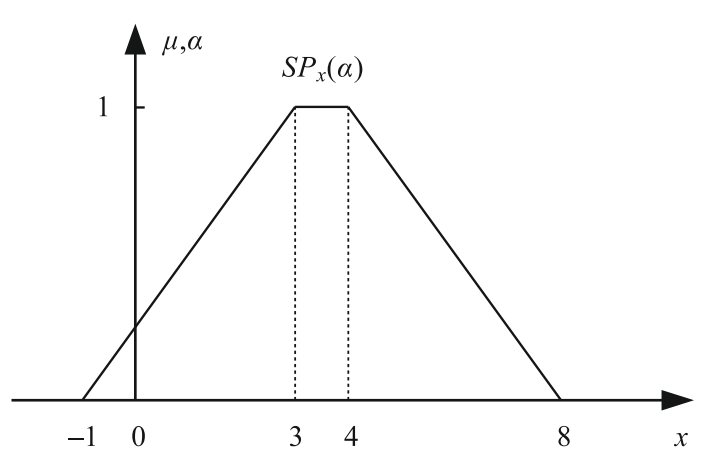

Fig. 10 Span $S P_{x}(\alpha)$ of the set $X\left(\alpha, \gamma_{a}, \gamma_{b}\right)$ of the result variable $x$ of the Eq. (20)
Formula (21) makes it possible to generate all possible states of the system realizing the dependence $a+x=b$ for each level of membership $\mu=\alpha$. For $\alpha=0$ possible states are e.g. $(a, b, x=b-a):(6,5,-1),(3,6,3),(1,5,4)$, $(1,9,8)$. In general, there are an infinite number of possible states $(a, b, x=b-a)$ satisfying the relationship $A+X=B$. Why was GIA based on gradual intervals unable to detect any of these states? This is due to the misconception that the correct span of the result variable $x$ can always be detected by $2 \mathrm{D}$ computations without finding a multidimensional solution, as MFA does. Kołodziejczyk et al. (2020) present an example of the use of HMF and MFA to solve system of equations of the order higher than 1 . The paper shows that MFA made it possible to find algebraic solutions in tasks where other kinds of fuzzy arithmetic have failed.

In the second example, a real object moving in uniformly decelerated motion will be considered. Let us assume a deceleration $a=-0.5 \frac{\mathrm{m}}{\mathrm{s}^{2}}$ and an initial velocity $V_{0}=22 \frac{\mathrm{m}}{\mathrm{s}}$. Our task will be to determine what distance the vehicle will travel after about 50s of movement.

Time of movement is defined imprecisely as 'about 50s' and we will define it using the triangular fuzzy number shown in Fig. 11. We can describe the time using HMF as: $t=40+10 \mu+\gamma_{t}(20-20 \mu), \quad \mu, \gamma_{t} \in[0,1]$.

The distance can be calculated from the formula: $d=0.5 a t^{2}+V_{0} t$.

After substituting time $t$, we have:

$$
\begin{aligned}
d\left(\mu, \gamma_{t}\right)= & 0.5 a\left(40+10 \mu+\gamma_{t}(20-20 \mu)\right)^{2} \\
& +V_{0}\left(40+10 \mu+\gamma_{t}(20-20 \mu)\right) .
\end{aligned}
$$

The solution $d\left(\mu, \gamma_{t}\right)$ is a function of two variables: $\mu$ and $\gamma_{t}$. Now, we can determine the span:

$$
\mathrm{SP}_{t}(\mu)=\left[\min _{\gamma_{t}} d\left(\mu, \gamma_{t}\right), \max _{\gamma_{t}} d\left(\mu, \gamma_{t}\right)\right], \quad \gamma_{t} \in[0,1]
$$

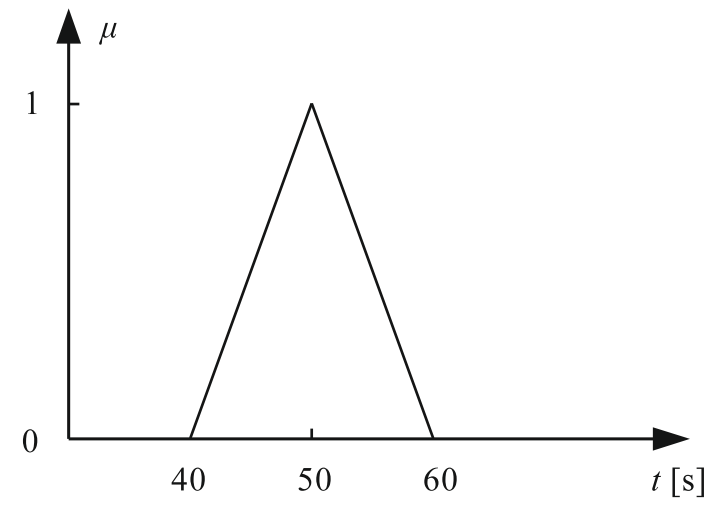

Fig. 11 Triangular fuzzy number defining time $t=$ 'about $50 \mathrm{~s}$ ' 
The plot of the span is presented in Fig. 12.

We can perform similar calculations using GIA. The time defined using InvMF has the form:

$t=[40+10 \mu, 60-10 \mu]$.

We can now determine formulas for the left and right borders of the resulting membership function, which is shown in Fig. 13.

$$
\begin{aligned}
& d^{-}(\mu)=0.5 a(40+10 \mu)^{2}+V_{0}(40+10 \mu) \\
& d^{+}(\mu)=0.5 a(60-10 \mu)^{2}+V_{0}(60-10 \mu)
\end{aligned}
$$

From the Fig. 13, we can see that the resulting fuzzy number determined using GIA is not consistent with the definition of a fuzzy number, unlike the result determined using MFA (Fig. 12). This is because MFA takes into account not only borders but also interiors of the fuzzy numbers in its calculations.

\section{Conclusions}

The paper presents a discussion with the view found among some fuzzy arithmetic researchers proclaiming the identity of the inverse membership function used in GIA and the horizontal membership function used in MFA. The similarity of InvMF and HMF also indirectly suggests the similarity (or even identity) of GIA and HMA. The paper shows that there are fundamental differences between InvMF (which is part of the gradual number used in GIA) and HMF. The gradual number is a mathematical object defined in 2D-space, and the InvMFs that are its elements (GN borders) are also 2D objects. In gradual arithmetic, arithmetic calculations are performed using boundaries of GNs. On the other hand, HMF is a mathematical object defined in 3D-space, including not only the borders but also

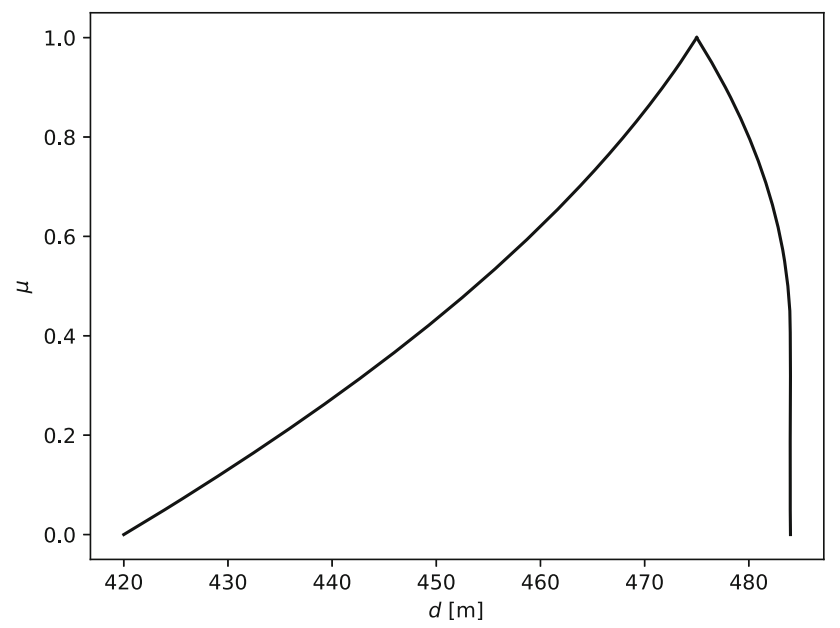

Fig. 12 Plot of the span determined with use of MFA

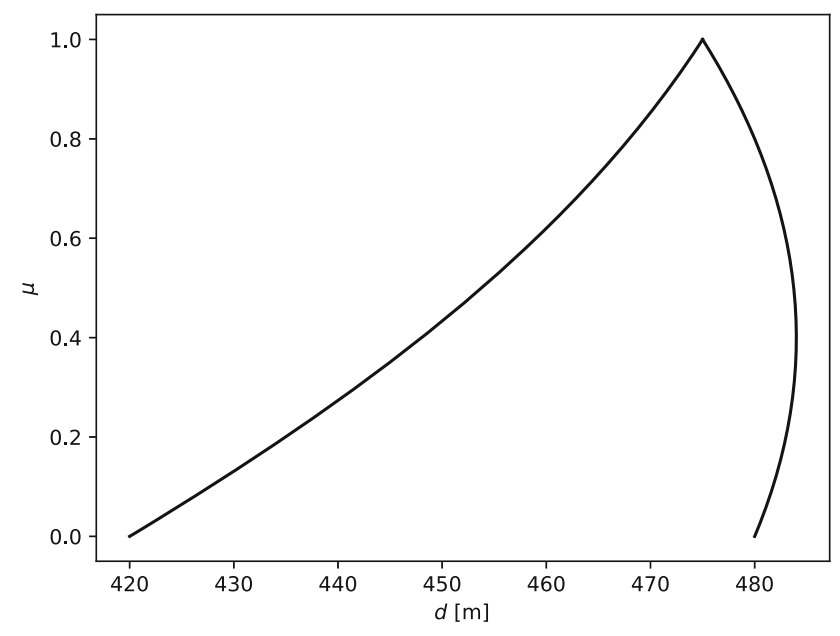

Fig. 13 Plot of the membership function determined with use of GIA

the interior of the membership function. This has a great impact on the computational results that are obtained.

$\mathrm{AFs}$, as models of borders of MF, are used in GIA to calculate the results separately, while HMF is a single overall model of MF which takes part in the calculations as one whole, without dividing it into parts. In general, this often leads to different computational results provided by GIs-based and HMFs-based fuzzy arithmetic types. First of all, the results of calculations in MFA are objects with dimensionality 3 or higher, while GIA always provides results with dimensionality 2 . This fact causes MFA to be able to provide, for example, algebraic solutions to equations that verify them after substitution. In the case of GIA, this is not always true, as shown by the simple example presented in the paper. The spans of multidimensional solutions provided by MFA are always consistent with the definition of a fuzzy number, while the solutions provided by GIA are sometimes inconsistent, as shown in the example and what is reported in the literature (Boukezzoula et al. 2021).

Open Access This article is licensed under a Creative Commons Attribution 4.0 International License, which permits use, sharing, adaptation, distribution and reproduction in any medium or format, as long as you give appropriate credit to the original author(s) and the source, provide a link to the Creative Commons licence, and indicate if changes were made. The images or other third party material in this article are included in the article's Creative Commons licence, unless indicated otherwise in a credit line to the material. If material is not included in the article's Creative Commons licence and your intended use is not permitted by statutory regulation or exceeds the permitted use, you will need to obtain permission directly from the copyright holder. To view a copy of this licence, visit http://creativecommons. org/licenses/by/4.0/. 


\section{References}

Boukezzoula R, Jaulin L, Foulloy L (2019) Thick gradual intervals: an alternative interpretation of type-2 fuzzy intervals and its potential use in type-2 fuzzy computations. Eng Appl Artif Intell 85:691-712

Boukezzoula R, Foulloy L, Coquin D et al (2021) Gradual interval arithmetic and fuzzy interval arithmetic. Granul Comput 6:451-471

Dubois D, Prade H (2008) Gradual elements in a fuzzy set. Soft Comput 12(2):165-175

Fortin J, Dubois D, Fargier H (2008) Gradual numbers and their application to fuzzy interval analysis. IEEE Trans Fuzzy Syst 16(2):388-402

Kołodziejczyk J, Piegat A, Sałabun W (2020) Which alternative for solving dual fuzzy nonlinear equations is more precise? Mathematics 8(9): 1507

Mazandarani M, Xiu L (2021) A review on fuzzy differential equations. IEEE Access 9:62195-62211

Mazandarani M, Zhao Y (2019) Z-differential equations. IEEE Trans Fuzzy Syst 28(3):462-473

Najariyan M, Zhao Y (2020) The explicit solution of fuzzy singular differential equations using fuzzy Drazin inverse matrix. Soft Comput 24(15):11251-11264
Piegat A, Landowski M (2013) Two interpretations of multidimensional RDM interval arithmetic: multiplication and division. Int J Fuzzy Syst 15(4):486-496

Piegat A, Landowski M (2015) Horizontal membership function and examples of its applications. Int J Fuzzy Syst 17(1):22-30

Piegat A, Landowski M (2021a) Multidimensional interval type 2 epistemic fuzzy arithmetic. Iran J Fuzzy Syst 18(5):19-26

Piegat A, Landowski M (2021b) Multidimensional type 2 epistemic fuzzy arithmetic based on the body definition of the type 2 fuzzy set. Appl Sci 11(13):5844

Piegat A, Pluciński M (2017) Fuzzy number division and the multigranularity phenomenon. Bull Pol Acad Sci Tech Sci 65(4):497-511

Piegat A, Pluciński M (2015) Fuzzy number addition with the application of horizontal membership functions. Sci World J:1-16 (Article ID: 367214)

Zadeh L (1965) Fuzzy sets. Inf Control 8:338-353

Zadeh L (1975) The concept of a linguistic variable and its application to approximate reasoning-I. Inf Sci 8(3):199-249

Publisher's Note Springer Nature remains neutral with regard to jurisdictional claims in published maps and institutional affiliations. 